Pacific Journal of Mathematics

SUPERREGULAR SEQUENCES 


\title{
SUPER-REGULAR SEQUENCES
}

\author{
JUdith D. SALLY
}

Let $(R, \underline{m})$ be a local ring with associated graded ring $g r R=R / \underline{m} \oplus \underline{m} / \underline{m}^{2} \oplus \underline{m}^{2} / \underline{m}^{3} \oplus \cdots$. This paper deals with the problem of finding properties of $R$ which lead to good properties in $g r R$. There are two main results in this paper which give techniques for recognizing when the maximal homogeneous ideal of $g r R$ contains regular elements. Applications of these results give examples of local CohenMacaulay rings which have Cohen-Macaulay associated graded rings.

Let $(R, \underline{m})$ be a local ring with associated graded ring $g r R=$ $R / \underline{m} \oplus \underline{m} / \underline{m}^{2} \oplus \underline{m}^{2} / \underline{m}^{3} \oplus \cdots$. Information about $g r R$ gives some measure of the singularity at $R$ since properties of $g r R$ yield data about the Hilbert function of $R$ and about monoidal transforms of $R$. However it is often difficult to compute $g r R$ and it is seldom true that properties of $R$ are carried over to $g r R$. Thus, it is important to recognize characteristics of $R$ which lead to good properties in $g r R$.

There are two main results in this paper which give techniques for recognizing good properties in $g r R$. The first shows when the initial forms of a regular sequence in $\mathrm{m} / \mathrm{m}^{2}$ form a regular sequence in $g r R$; the given regular sequence is then called super-regular. (All our applications use homogeneous regular sequences of degree one in $g r R$, so we have avoided some complications by primarily considering this case. If $R / \underline{m}$ is infinite and if $g r \underline{m}$ contains a regular sequence of length $t$, it contains a homogeneous regular sequence of degree one of length $t$.) The second result shows that for certain local Cohen-Macaulay rings the question of whether $g r R$ is Cohen-Macaulay can be reduced to the same question for such Cohen-Macaulay rings of dimension one. The paper concludes with several applications of these results. The applications give examples of local Cohen-Macaulay rings which have Cohen-Macaulay associated graded rings.

We begin with some definitions. For any nonzero element $x$ in the local ring $(R, \underline{m})$, let $\bar{x}$ denote the initial form of $x$ in $g r R$, i.e., if $x \in \underline{m}^{s} \backslash \underline{m}^{s+1}, \bar{x}=x+\underline{m}^{s+1} \in \underline{m}^{s} / \underline{m}^{s+1}$. We will say that $x$ has order $s$ and that $\bar{x}$ has degree $s$. Let $\overline{0}$ be the zero element in $g r R$. 0 has infinite order and $\overline{0}$ has infinite degree. A system of elements of $R$ (of $g r R$ ) has order $s$ (degree $s$ ) if each element has order $s$ (degree $s$ ). 
Recall that if $S$ is any commutative ring, a sequence of elements $x_{1}, \cdots, x_{t}$ of $S$ is a regular sequence if $\left(x_{1}, \cdots, x_{t}\right) S \neq S$ and if, for $i=1, \cdots, t, x_{i}$ is not a zero divisor on $S /\left(x_{1}, \cdots, x_{i-1}\right) S$.

For the case of a local ring $(R, m)$ it is important to know when $g r m$, the maximal homogeneous ideal of $g r R$, contains a regular element. For the existence of a regular element in $\mathrm{grm}$ permits a change of rings which reduces dimension. This is described in the following lemma.

Lemma 0.1. Let $(R, \underline{m})$ be a local ring with associated graded ring grR. Suppose that grm, the maximal homogeneous ideal of grR, contains a regular element. Then grm contains a homogeneous regular element $\bar{x}$ and, if $x$ is any element of $R$ with initial form $\bar{x}$, there is an isomorphism of graded rings $g r R / \bar{x} g r R \cong g r(R / x R)$ induced by the natural map of local rings $R \rightarrow R / x R$.

Proof. By [8, Ch. VII, §2], the prime ideals $P_{1}, \cdots, P_{h}$ in Ass $g r R$ are homogeneous. By [8, pg. 286, footnote], grm $\nsubseteq \cup \cup P_{i}$ implies that there is a homogeneous element $\bar{x}$ of positive degree $s$, say, such that $\bar{x} \notin P_{i}$ for $i=1, \cdots, h$. Let $x$ be any element of $R$ with initial form $\bar{x}$. Consider the natural homomorphism $\nu: R \rightarrow R / x R$. Since $\nu\left(m^{n}\right) \subseteq(m / x R)^{n}$, the induced homomorphism $g r \nu: g r R \rightarrow g r(R / x R)$ given by $(g r \nu)_{n}: \underline{m}^{n} / \underline{m}^{n+1} \rightarrow(\underline{m} / x R)^{n} /(\underline{m} / x R)^{n+1}$ is a homomorphism of graded rings, ef. [1, Ch. 3, $\S 2$, no. 4]. To show that grv is surjective with kernel $\bar{x} g r R$, it is enough to show that $(g r \nu)_{n}$ is surjective with kernel $(\bar{x} g r R)_{n}$. It is clear that $(g r \nu)_{n}$ is surjective. If $\bar{w} \in \underline{m}^{n} / \underline{m}^{n+1}$ and $(g r \nu)_{n} \bar{w}=0$, then $w \in\left(\underline{m}^{n+1}+x R\right) \cap \underline{m}^{n}=\underline{m}^{n+1}+$ $\left(x R \cap \underline{m}^{n}\right)$ for any $w \in \bar{w}$. Since $\bar{x}$ is regular in $g r R, x R \cap \underline{m}^{n}=x \underline{m}^{n-1}$, where a nonpositive power of $\underline{m}$ is understood to be $R$. Thus $w=z+x y$ with $z \in \underline{m}^{n+1}$ and $y \in \underline{m}^{n-s}$. Again using that $\bar{x}$ is regular in $g r R$, we have that $\bar{x} \bar{y}=\overline{x y}=\overline{z+x y}=\bar{w}$. This proves that $\bar{w} \in$ $\bar{x} g r R$ and completes the proof of the lemma.

We will say that an element $x$ in $\underline{m}$, the maximal ideal of a local ring $(R, \underline{m})$, is super-regular if $\bar{x}$ is a regular element in $g r R$. A sequence of elements $x_{1}, \cdots, x_{t}$ in $R$ will be called a super-regular sequence if $\bar{x}_{1}, \cdots, \bar{x}_{t}$ is a regular sequence in $g r R$.

Several properties of super-regular sequences follow easily. A super-regular sequence $x_{1}, \cdots, x_{t}$ is a regular sequence. For if $\bar{x}_{1}$ is a regular element in $g r \underline{m}, x_{1}$ is a regular element in $R$ and, by (0.1), $g r\left(R / x_{1} R\right) \cong g r R / \bar{x}_{1} g r R$. By induction, the images of $x_{2}, \cdots, x_{t}$ form a regular sequence in $R / x_{1} R$ so $x_{1}, x_{2}, \cdots, x_{t}$ is a regular sequence in $R$. A similar isomorphism shows that a sequence $x_{1}, \cdots, x_{t}$ of elements of $R$ is super-regular if and only if there is some $j, 1 \leqq j \leqq t$, such that $x_{1}, \cdots, x_{j}$ is super-regular in $R$ and 
the image of $x_{j+1}, \cdots, x_{t}$ is super-regular in $R /\left(x_{1}, \cdots, x_{j}\right) R$. It is also true that any permutation of a super-regular sequence is superregular.

In addition to the notation and definitions introduced above, the following notation will be used. $\lambda(A)=\lambda_{R}(A)$ denotes the length of an $R$-module $A$. If $I$ is an ideal in a local $\operatorname{ring}(R, \underline{m}), v(I)$ denotes the number of generators in a minimal basis of $I . \quad v(\underline{m})$ is the embedding dimension of $R . \quad e=e(R)$ is the multiplicity of $R$. We will often use $\underline{x}$ to denote a system of elements $x_{1}, \cdots, x_{t}$ in $R$.

Let $\underline{x}=x_{1}, \cdots, x_{t}$ be a regular sequence in $(R, \underline{m})$. If we say that $f\left(x_{1}, \cdots, x_{t}\right)$ is a form of degree $s$ in $x_{1}, \cdots, x_{t}$, we mean that $f\left(X_{1}, \cdots, X_{t}\right)$ is a degree $s$ homogeneous polynomial in the polynomial ring $R\left[X_{1}, \cdots, X_{t}\right]$ and $f\left(x_{1}, \cdots, x_{d}\right)$ is the image of $f\left(X_{1}, \cdots, X_{t}\right)$ in $R$ under the homomorphism sending $X_{i}$ to $x_{i}$.

We will use the notion of minimal reduction of the maximal ideal of a local ring $(R, \underline{m})$, cf. [3], but we wish to take a more restrictive definition than in [3]. If $(R, \underline{m})$ is a $d$-dimensional local ring, a system $x=x_{1}, \cdots, x_{d}$ of $d$ elements of $R$ is a minimal reduction of $\underline{m}$ if there is a positive integer $r$ such that $\underline{m}^{r+1}=\underline{x} \underline{m}^{r}$. If $R / \underline{m}$ is infinite, minimal reductions exist, as a minimal reduction of $\underline{m}$ is the preimage in $R$ of a degree one homogeneous system of parameters in $g r R$. The existence of a minimal reduction is hardly ever a troublesome hypothesis because the change of rings $R \rightarrow$ $R(U)=R[U]_{m_{R[U]}}, U$ an indeterminate, is faithfully flat and $\underline{m} R(U)$ has minimal reductions.

1. When are regular sequences super-regular? If $x$ is a regular element of order one in the local ring $(R, \underline{m})$, it is clear that $x$ is super-regular if and only if $(x) \cap \underline{m}^{i+1}=x \underline{m}^{i}$ for all $i \geqq 0$. The assumption that $x$ is regular cannot be dropped as the example $k[[x, y]] /\left(y^{2}, x y\right), k$ a field, shows. An analogous characterization holds for a regular sequence $\underline{x}=x_{1}, \cdots, x_{t}$ of order one. It will be useful to prove a little more. We will see that $l$ intersection equalities $(\underline{x}) \cap \underline{m}^{i+1}=\underline{x} \underline{m}^{i}$ for $i \leqq l$ means that $\underline{x}$ is super-regular "up to $\underline{m}^{l+1}$."

THeOREM 1.1. Let $(R, \underline{m})$ be a local ring and let $\underline{x}=x_{1}, \cdots, x_{t}$ be a regular sequence of order one. Then,

$$
(\underline{x}) \cap \underline{m}^{i+1}=\underline{x}^{i},
$$

for all positive integers $i \leqq$ some positive integer $l$ if and only if

$$
\left(\left(\bar{x}_{1}, \cdots, \bar{x}_{j-1}\right): \bar{x}_{j}\right) \leqq\left(\bar{x}_{1}, \cdots, \bar{x}_{j-1}\right)+(g r \underline{m})^{l}
$$

for $1 \leqq j \leqq t$. 
We first note the following.

Lemma 1.2. Let $(R, \underline{m})$ be a local ring and let $\underline{x}=x_{1}, \cdots, x_{t}$ be a regular sequence of order one. If for all positive integers $i \leqq$ some positive integer $l,(\underline{x}) \cap \underline{m}^{i+1}=\underline{x}^{i}$, then

$$
(\underline{x})^{j} \cap \underline{m}^{i+1}=(\underline{x})^{j} \underline{m}^{i+1-j},
$$

for $1 \leqq j \leqq i+1$ and $i \leqq l$.

Proof. We prove the lemma by induction on $j$. Let $j>1$. Let $f_{j}\left(x_{1}, \cdots x_{t}\right)$, a form of degree $j$ in $x_{1}, \cdots, x_{t}$, be in $(\underline{x})^{j} \cap \underline{m}^{i+1} \subset$ $(\underline{x})^{j-1} \cap \underline{m}^{i+1}=\underline{x}^{j-1} \underline{m}^{i+1-j+1} . \quad f_{j}\left(x_{1}, \cdots, x_{t}\right)=g_{j-1}\left(x_{1}, \cdots, x_{t}\right)$, where $g_{j-1}$ is a form of degree $j-1$ in $x_{1}, \cdots, x_{t}$ with coefficients in $m^{i+1-j+1}$. Since $\left(x_{1}, \cdots, x_{t}\right)^{j-1} /\left(x_{1}, \cdots, x_{t}\right)^{j}$ is free over $R /\left(x_{1}, \cdots, x_{t}\right) R$, the coefficients of $g_{j-1}$ are in $(\underline{x}) \cap \underline{m}^{i+1-j+1}=\underline{x} \underline{m}^{i+1-j}$, so $f_{j}\left(x_{1}, \cdots, x_{t}\right) \in$ $(\underline{x}) \underline{m}^{j i+1-j}$.

Proof of Theorem 1.1. Assume that $(\underline{x}) \cap \underline{m}^{i+1}=\underline{x} \underline{m}^{i}$ for $i \leqq l$. We will prove that $\left(\left(\bar{x}_{1}, \cdots, \bar{x}_{j-1}\right): \bar{x}_{i}\right) \subseteq\left(\bar{x}_{1}, \cdots, \bar{x}_{j-1}\right)+(g r \underline{m})^{l}$ for $1 \leqq j \leqq t$, by induction on $t$. The proof for $t=1$ is immediate so we will assume that $t>1$. First, we show that $\left(0: \bar{x}_{1}\right) \leqq(g r \underline{m})^{l}$, i.e., that $x_{1} z \in \underline{m}^{i+1}$ implies that $z \in \underline{m}^{i}$ for $i \leqq l$. If $x_{1} z \in \underline{m}^{2}$, then $x_{1} z=$ $x_{1} a_{1}+\cdots+x_{t} a_{t}$ with $a_{1}, \cdots, a_{t} \in \underline{m}$. Thus $z-a_{1} \in\left(x_{2}, \cdots, x_{t}\right)$ and $z \in \underline{m}$. We claim that the following relation holds:

( * $\quad\left(m^{i+1}: x_{1}\right) \cap(\underline{x})^{j} \underline{m} \subseteq(\underline{x})^{j} \underline{m}^{i+1-j-1}+(\underline{x})^{j+1} \underline{m}$,

for $2 \leqq i \leqq l$ and $0 \leqq j \leqq i-2$. Suppose $\left(^{*}\right)$ has been proved. Let $x_{1} z_{0} \in \underline{m}^{i+1}$ for $i \geqq 2$. Then, with $j=0$ in $(*)$, we get $z_{0} \in \underline{m}^{i}+\underline{x} \underline{m}$. $z_{0}=\mu_{i}+z_{1}$ with $\mu_{i} \in \underline{m}^{i}$ and $z_{1} \in\left(\underline{m}^{i+1}: x_{1}\right) \cap \underline{x} \underline{m}$. Now we apply (*) with $j=1$ and continue in this way until we have $z_{0}=\tilde{\mu}_{i}+z_{i-2}$ with $\tilde{\mu}_{i} \in \underline{m}^{i}$ and $z_{i-2} \in\left(\underline{m}^{i+1}: x_{1}\right) \cap(\underline{x})^{i-2} \underline{m}$. Finally, we apply $\left(^{*}\right)$ with $j=i-2$, to get $z_{i-2} \in(\underline{x})^{i-2} \underline{m}^{2}+(\underline{x})^{i-1} \underline{m} \cong \underline{m}^{i}$.

Now we prove $\left({ }^{*}\right)$. Let $j=0$. Let $x_{1} z \in \underline{m}^{i+1}$ with $i \geqq 2 . \quad x_{1} z \in$ $\underline{m}^{i+1} \cap(\underline{x})=\underline{x} \underline{m}^{i}$, so $x_{1} z=a_{1} x_{1}+\cdots+a_{t} x_{t}$ with $a_{1}, \cdots, a_{t} \in \underline{m}^{i}$. $z-$ $a_{1} \in\left(x_{2}, \cdots, x_{t}\right)$ since $x_{1}, \cdots, x_{t}$ is a regular sequence. In fact, $z-a_{1} \in$ $\left(x_{2}, \cdots, x_{t}\right) \underline{m}$. For $\left(z-a_{1}\right) x_{1} \in\left(x_{2}, \cdots, x_{t}\right) x_{1} \cap \underline{m}^{i+1} \subseteq(\underline{x})^{2} \cap \underline{m}^{i+1}=(\underline{x})^{2} \underline{m}^{i-1}$ by (1.2).

Assume that $j>0 . \quad$ Let $z \in\left(\underline{m}^{i+1}: x_{1}\right) \cap(\underline{x})^{j} \underline{m} . \quad z=g_{j}\left(x_{1}, \cdots, x_{t}\right)$ is a homogeneous polynomial in $x_{1}, \cdots, x_{t}$ of degree $j$ with coefficients in $\underline{m}$. $z x_{1} \in \underline{m}^{i+1} \cap(\underline{x})^{j+1}=(\underline{x})^{j+1} \underline{m}^{i+1-j-1}$ by $(1.2)$, so

$$
z x_{1}=g_{j}\left(x_{1}, \cdots, x_{t}\right) x_{1}=h_{j+1}\left(x_{1}, \cdots, x_{t}\right)
$$

with $h_{j+1}$ a homogeneous polynomial in $x_{1}, \cdots, x_{t}$ of degree $j+1$ 
and coefficients in $\underline{m}^{i+1-j-1}$. Equating coefficients of like monomials of degree $j+1$ in $\left(^{* *}\right)$, we see that the coefficients of $g_{j}\left(x_{1}, \cdots, x_{t}\right)$ are in $\underline{m}^{i+1-j-1}+(\underline{x})$. Thus $g_{j}\left(x_{1}, \cdots, x_{t}\right)=\xi+f_{j+1}\left(x_{1}, \cdots, x_{t}\right)$ with $\xi \in(\underline{x})^{j} \underline{m}^{i+1-j-1}$ and $f_{j+1}\left(x_{1}, \cdots, x_{t}\right) \in(\underline{x})^{j+1}$. But $x_{1}(\underline{x})^{j+1} \cap \underline{m}^{i+1} \subseteq(\underline{x})^{j+2} \cap$ $\underline{m}^{i+1}=(\underline{x})^{j+2} \underline{m}^{i+1-j-2}$ by (1.2). Consequently, $\left(g_{j}\left(x_{1}, \cdots, x_{t}\right)-\xi\right) x_{1}=$ $f_{j+1}\left(x_{1}, \cdots, x_{t}\right) x_{1} \in(\underline{x})^{j+2} \underline{m}^{i+1-j-2}$ and $f_{j+1}\left(x_{1}, \cdots, x_{t}\right) \in(\underline{x})^{j+1} \underline{m}$. Thus $g_{j}\left(x_{1}, \cdots, x_{t}\right) \in(\underline{x})^{j} \underline{m}^{i+1-j-1}+(\underline{x})^{j+1} \underline{m}$. This completes the proof that $\left(0: \bar{x}_{1}\right) \subseteq(g r \underline{m})^{l}$.

Pass to $(\widetilde{R}, \underline{\tilde{m}})=\left(R / x_{1} R, \underline{m} / x_{1} R\right)$. The images $\widetilde{x}_{2}, \cdots, \widetilde{x}_{t}$ form a regular sequence in $\widetilde{R}$ and $\underline{\widetilde{m}}^{i+1} \cap\left(\widetilde{x}_{2}, \cdots, \widetilde{x}_{t}\right) \widetilde{R}=\left(\widetilde{x}_{2}, \cdots, \widetilde{x}_{t}\right) \underline{\tilde{m}}^{i}$ for $i \leqq l$. By induction on $t$, we have that $\left(\left(\overline{\widetilde{x}}_{2}, \cdots, \overline{\widetilde{x}}_{j-1}\right): \overline{\widetilde{x}}_{j}\right) \subseteq$ $\left(\overline{\widetilde{x}}_{2}, \cdots, \overline{\widetilde{x}}_{j-1}\right)+(g r \underline{\tilde{m}})^{l} \cong\left(\bar{x}_{1}, \bar{x}_{2}, \cdots, \bar{x}_{j-1}\right) g r R / \bar{x}_{1} g r R+\left((g r \underline{m})^{l}, \bar{x}_{1}\right) g r R / \bar{x}_{1} g r R$. Thus, $\left(\left(\bar{x}_{1}, \cdots, \bar{x}_{j-1}\right): \bar{x}_{j}\right) \subseteq\left(\bar{x}_{1}, \cdots, \bar{x}_{j-1}\right)+(g r \underline{m})^{l}$.

For the converse we assume that $x_{1}, \cdots, x_{t}$ is a regular sequence in $R$ with $\left(\left(\bar{x}_{1}, \cdots, \bar{x}_{j-1}\right): \bar{x}_{j}\right) \leqq\left(\bar{x}_{1}, \cdots, \bar{x}_{j-1}\right)+(g r \underline{m})^{l}$ for $1 \leqq j \leqq t$ and again use induction on $t$. We have $\left(0: \bar{x}_{1}\right) \subseteq(\operatorname{grm})^{l}$ so $\left(x_{1}\right) \cap \underline{m}^{i+1}=$ $x_{1} \underline{m}^{i}$ for $i \leqq l$. The proof is finished if $t=1$ so we take $t>1$. We have $\left(g r R / \bar{x}_{1} g r R\right)_{j} \cong\left(g r\left(R / x_{1} R\right)\right)_{j}$ for $j=0, \cdots, l+1$ since

$$
\left(g r R / \bar{x}_{1} g r R\right)_{j}=\underline{m}^{j} / x_{1} \underline{m}^{j-1}+\underline{m}^{j+1}
$$

and $\left(g r\left(R / x_{1} R\right)\right)_{j}=\left(\underline{m}^{j}, x_{1}\right) /\left(\underline{m}^{j+1}, x_{1}\right) \cong \underline{m}^{j} / \underline{m}^{j+1}+\left(x_{1}\right) \cap \underline{m}^{j}$. This means that the required hypotheses are satisfied by $\widetilde{x}_{2}, \cdots, \widetilde{x}_{t}$, the images of $x_{2}, \cdots, x_{t}$ in $(\widetilde{R}, \widetilde{m})=\left(R / x_{1} R, \underline{m} / x_{1} R\right)$. By induction on $t$ we have $\left(\widetilde{x}_{2}, \cdots, \widetilde{x}_{t}\right) \cap \underline{\tilde{m}}^{i+1}=\left(\widetilde{x}_{2}, \cdots, \widetilde{x}_{t}\right) \underline{\tilde{m}}^{j}$ for $i \leqq l$, so that $\left(x_{1}, \cdots, x_{t}\right) \cap$ $\left(\underline{m}^{i+1}, x_{1}\right)=\left(x_{1}, \cdots, x_{t}\right) \underline{m}^{i}+x_{1} R$. Let $w \in\left(x_{1}, \cdots, x_{t}\right) \cap m^{i+1} . \quad w=$ $a x_{1}+\mu_{2} x_{2}+\cdots+\mu_{t} x_{t}$ with $\mu_{2}, \cdots, \mu_{t} \in \underline{m}^{i}$. Then $a x_{1} \in \underline{m}^{i+1}$ so $a \in \underline{m}^{i}$ and $w \in\left(x_{1}, \cdots, x_{t}\right) \underline{m}^{i}$.

CoRollary 1.3. Let $\underline{x}=x_{1}, \cdots, x_{t}$ be a regular sequence in a local ring $(R, \underline{m})$ such that $\left(x_{1}, \cdots, x_{t}\right) \cap \underline{m}^{i+1}=\left(x_{1}, \cdots, x_{t}\right) \underline{m}^{i}$ for $i \leqq l$. Then $\left(x_{1}, \cdots, x_{s}\right) \cap \underline{m}^{i+1}=\left(x_{1}, \cdots, x_{s}\right) \underline{m}^{i}$ for $1 \leqq s \leqq t$ and $0 \leqq i \leqq l$.

Corollary $1.4 .^{1}$ Let $(R, \underline{m})$ be a local ring and let $\underline{x}=x_{1}, \cdots, x_{t}$ be a regular sequence. $x_{1}, \cdots, x_{t}$ is super-regular if and only if $(\underline{x}) \cap \underline{m}^{i+1}=\underline{x}^{i}$ for all $i \geqq 0$.

Proof. If $(\underline{x}) \cap \underline{m}^{i+1}=\underline{x}^{i}$ for all $i \geqq 0$, then, by (1.1),

$$
\left(\left(\bar{x}_{1}, \cdots, \bar{x}_{j-1}\right): \bar{x}_{j}\right) \subseteq \bigcap_{l=0}^{\infty}\left(\bar{x}_{1}, \cdots, \bar{x}_{j-1}\right)+(g r \underline{m})^{l}=\left(\bar{x}_{1}, \cdots, \bar{x}_{j-1}\right) .
$$

The converse also follows immediately from (1.1).

1 Added in proof. Corollary 1.4 is a special case of Corollary 2.7 of the paper "Form rings and regular sequences" by P. Valabrega and G. Valla which recently appeared in Nagaya Math. J., 72 (1978), 93-101. 
Corollary 1.5. Let $(R, \underline{m})$ be a d-dimensional local CohenMacaulay ring. $g r R$ is Cohen-Macaulay if there is a minimal reduction $\underline{x}=x_{1}, \cdots, x_{d}$ of $\underline{m}$ such that $(\underline{x}) \cap \underline{m}^{i+1}=\underline{x} \underline{m}^{i}$ for all $i \geqq 0$.

Proof. By (1.4), $\bar{x}_{1}, \cdots, \bar{x}_{d}$ is a homogeneous system of parameters which is a regular sequence. By [2], $g r R$ is Cohen-Macaulay.

REMARK. (1.5) is similar to a special case of Theorem 4.4 in [2].

We take a moment to note the connection between superregularity and the notion of analytic independence in $\underline{m}$. Recall, [3], that a system of elements $\underline{x}=x_{1}, \cdots, x_{t}$ in a local ring $(R, \underline{m})$ is analytically independent in $m$ if given $f_{s}\left(X_{1}, \cdots, X_{t}\right)$, a homogeneous polynomial in $R\left[X_{1}, \cdots, X_{t}\right]$ of (arbitrary) degree $s$, such that $f_{s}\left(x_{1}, \cdots, x_{t}\right) \in \underline{m}^{s+1}$ then all the coefficients of $f_{s}$ are in $\underline{m}$. Stated differently, $\underline{x}=x_{1}, \cdots, x_{t}$ is analytically independent in $\underline{m}$ if $\left(x_{1}, \cdots, x_{t}\right)^{s} \cap \underline{m}^{s+1}=\left(x_{1}, \cdots, x_{t}\right)^{s} m$ for all positive integers $s$. Note also that if a regular sequence $\underline{x}=x_{1}, \cdots, x_{t}$ in $(R, \underline{m})$ satisfies $(\underline{x}) \cap \underline{m}^{i+1}=\underline{x} \underline{m}^{i}$ for $i \leqq l$ and if $f_{s}\left(X_{1}, \cdots, X_{t}\right)$ is a form of degree $s$ in $R\left[X_{1}, \cdots, X_{t}\right]$ with $f_{s}\left(x_{1}, \cdots, x_{t}\right) \in \underline{m}^{i+1}$ for $i \leqq l$, then all one can say about the coefficients of $f_{s}$ is that they are in $\left(\underline{m}^{i+1-s}, x_{1}, \cdots, x_{t}\right)$ as the example $f\left(X_{1}, X_{2}\right)=x_{2} X_{1}-x_{1} X_{2}$ shows.

Next we give a direct proof that intersection equalities as in (1.1) give information about minimal bases for powers of $\underline{m}$. This can also be done using the Hibert sum transform as in [7]. The idea is that $(\underline{x}) \cap \underline{m}^{i+1}=\underline{x} \underline{m}^{i}$ for $i \leqq l$ implies that multiplication of $\underline{m}^{i} / \underline{m}^{i+1}$ by each $\bar{x}_{j}$, for $x_{j}$ in the regular sequence $x$, is a monomorphism for $i \leqq l$.

We introduce some notation. Let $\underline{x}=x_{1}, \cdots, x_{t}$ be a regular sequence in the local ring $(R, \underline{m})$. Let $\mathscr{H}_{i}(\underline{x})$ denote the set of monomials of degree $i$ in $x_{1}, \cdots, x_{t}$. If $J$ is a set of elements of $R$, $\mathscr{K}_{i}(\underline{x}) J$ denotes the set of products $\left\{\xi j \mid \xi \in \mathscr{K}_{i}(\underline{x}), j \in J\right\}$.

Theorem 1.6. Let $(R, \underline{m})$ be a local ring and let $\underline{x}=x_{1}, \cdots, x_{t}$ be a regular sequence such that $x_{1}, \cdots, x_{t}, z_{t+1}, \cdots, z_{v}$ is a minimal basis for $\underline{m}$. Suppose that $(\underline{x}) \cap \underline{m}^{i+1}=\underline{x}^{i}$ for $i \leqq l$. Then, for each $i \leqq l$, the set $\mathscr{K}_{i}(\underline{x}) \cup \mathscr{K}_{i-1}(\underline{x}) \mathscr{Z}_{1} \cup \cdots \cup \mathscr{H}_{1}(\underline{x}) \mathscr{K}_{i-1}$ is a subset of a minimal basis for $\underline{m}^{i}$, where $\mathscr{F}_{j}$ is the set of monomials in $z_{t+1}, \cdots, z_{v}$ needed to complete the set $\mathscr{K}_{j}(\underline{x}) \cup \cdots \mathscr{K}_{1}(\underline{x}) \mathscr{Y}_{j-1}$ to a minimal basis of $\underline{m}^{j}$.

Proof. We prove the theorem by induction on $i$ and $t$. We may assume $i>1$. Let $t=1$. If

$$
a x_{1}^{i}+b x_{1}^{i-1} \xi_{11}+\cdots+c x_{1} \xi_{i-1 n_{i-1}} \in \underline{m}^{i+1}
$$


where $\xi_{p q}, 1 \leqq p \leqq i-1,1 \leqq q \leqq n_{p}$, are the monomials $z_{t+1}, \cdots, z_{v}$ completing the basis for $\underline{m}^{p}$, then $x_{1}\left(a x_{1}^{i-1}+b x_{1}^{i-2} \xi_{11}+\cdots+c \xi_{i-1 n_{i-1}}\right) \in$ $\underline{m}^{i+1}$. Thus, $a x_{1}^{i-1}+b x_{1}^{i-2} \xi_{11}+\cdots+c \xi_{i-1 n_{i-1}} \in \underline{m}^{i}$ so, by induction on $i$, the coefficients $a, b, \cdots, c$ are in $m$. Assume that $t>1$. Let $\widetilde{x}_{2}, \cdots, \widetilde{x}_{t}$, $\widetilde{z}_{t \pm 1}, \cdots, \widetilde{z}_{v}$ be the images of $x_{2}, \cdots, x_{t}, z_{t+1}, \cdots, z_{v}$ in $(\widetilde{R}, \underline{\widetilde{r}})=$ $\left(R / x_{1} R, m / x_{1} R\right)$. Note that for $j<i$ we have that

$$
\mathscr{K}_{j}\left(\widetilde{x}_{2}, \cdots, \widetilde{x}_{t}\right) \cup \mathscr{K}_{j-1}\left(\widetilde{x}_{2}, \cdots, \widetilde{x}_{t}\right) \tilde{\mathscr{X}}_{1} \cup \cdots \cup \mathscr{K}_{1}\left(\widetilde{x}_{2}, \cdots, \widetilde{x}_{t}\right) \tilde{\mathscr{X}}_{j-1} \cup \tilde{\mathscr{x}}_{j}
$$

is a minimal basis for $\widetilde{m}^{j}$. This set is the image in $\widetilde{R}$ of all the monomials in the chosen minimal basis for $\underline{m}^{j}$ which do not contain $x_{1}$. It is clear that this set is a generating set for $\tilde{m}^{j}$. It is minimal because

$$
\begin{aligned}
\operatorname{dim}_{R / m} \underline{\tilde{m}}^{j} / \underline{\tilde{m}}^{j+1} & =\operatorname{dim}_{R / m} \underline{m}^{j} / m^{j+1}+x_{1} \underline{m}^{j-1} \\
& =\operatorname{dim}_{R / m} \underline{m}^{j} / \underline{m}^{j+1}-\operatorname{dim}_{R / m} x_{1} \underline{m}^{j-1}+\underline{m}^{j+1} / \underline{m}^{j+1}
\end{aligned}
$$

and $\operatorname{dim}_{R / m} x_{1} \underline{m}^{j-1}+\underline{m}^{j+1} / \underline{m}^{j+1}$ is the number of monomials in the chosen basis for $m^{j}$ which contain $x_{1}$.

Suppose that

$$
\text { (*) } \quad a x_{1}^{\alpha_{1}} \cdots x_{t}^{\alpha_{t}}+\cdots+b x_{1}^{\beta_{1}} \cdots x_{t}^{\beta^{t}} \xi_{11}+\cdots+c x_{t} \xi_{\imath-1 n_{i-1}} \in \underline{m}^{i+1}
$$

is a relation $\bmod \underline{m}^{2+1}$ among the elements of the set $\mathscr{L}_{i}(\underline{x}) \cup$ $\mathscr{K}_{i-1}^{2}(\underline{x}) \mathscr{Y}_{1} \cup \cdots \cup \mathscr{K}_{1}(\underline{x}) \mathscr{Y}_{i-1}$. By passing to $\widetilde{R}$ and using induction we get that all the coefficients of monomials in $\left(^{*}\right)$ which do not contain $x_{1}$ are in $m$. So we have a relation

$$
a x_{1}^{\alpha_{1}} \cdots x_{t}^{\alpha_{t}}+\cdots+b x_{1}^{\beta_{1}} \cdots x_{t}^{\beta} t \xi_{11}+\cdots+c x_{1} \xi_{i-1 n_{i-1}} \in \underline{m}^{i+1}
$$

with $x_{1}$ appearing in each monomial. Thus,

$$
x_{1}\left(a x_{1}^{\alpha_{1}-1} \cdots x_{t}^{\alpha_{t}}+\cdots+b x_{1}^{\beta_{1}-1} \cdots x_{t}^{\beta} \xi_{11}+\cdots+c_{\xi_{2-1 n_{i-1}}}\right) \in \underline{m}^{i+1} .
$$

By (1.3), $\left(x_{1}\right) \cap \underline{m}^{i+1}=x_{1} \underline{m}^{i}$ for $i \leqq l$, so

$$
a x_{1}^{\alpha_{1}-1} \cdots x_{t}^{\alpha_{l}}+\cdots+b x_{1}^{\beta_{1}-1} \cdots x_{t}^{\beta} \xi_{11}+\cdots+c \xi_{i-1 n_{i-1}} \in \underline{m}^{i} .
$$

By induction, all the coefficients $a, b, \cdots, c$ are in $\underline{m}$.

ExAmple. We illustrate (1.6) with an example. Let $R$ be the ring $k\left[\left[t^{7}, t^{8}, t^{13}, t^{19}\right]\right]$ with $k$ a field. $\quad \underline{m}=\left(t^{7}, t^{8}, t^{13}, t^{19}\right) . \quad\left(t^{7}\right) \cap m^{i+1}=t^{7} m^{i}$ for $i=1,2,3$ but $\left(t^{7}\right) \cap \underline{m}^{5} \supseteqq t^{7} \underline{m}^{4}$ as $t^{40}=t^{7} \cdot t^{7}\left(t^{13}\right)^{2}$ is in $\underline{m}^{5}$ but $t^{7}\left(t^{13}\right)^{2}$ is not in $m^{4}$. We can apply (1.6) to get that $t^{7}\left\{t^{7}, t^{8}, t^{13}, t^{19}\right\}$ is part of a minimal basis for $m^{2}=\left(t^{7} m, t^{16}\right)$ and $t^{7}\left\{t^{14}, t^{15}, t^{20}, t^{26}, t^{16}\right\}$ is part of a minimal basis for $\underline{m}^{3}=\left(t^{7} \underline{m}^{2}, t^{24}\right)$.

2. Reduction to lower dimension. Let $(R, \underline{m})$ be a $d$-dimen- 
sional local Cohen-Macaulay ring of multiplicity $e$ and embedding dimension $v$. If $v=d, d+1$ or $e+d-1$ then $g r R$ is CohenMacaulay, cf. [4]. The property $\mathscr{P}_{k}$ for a local Cohen-Macaulay ring to have embedding dimension $e+d-k$ is preserved under passage to $R(U)=R[U]_{\underline{m} R[U]}, U$ an indeterminate, and is preserved under reduction modulo the ideal generated by any element of minimal reduction of $\underline{m}$. Given a local Cohen-Macaulay ring with a property $\mathscr{P}$ which is preserved under both types of change of rings mentioned above, (2.4) below shows that if every 1-dimensional local CohenMacaulay ring with $\mathscr{P}$ has Cohen-Macaulay associated graded ring then so does every $d$-dimensional local Cohen-Macaulay ring with $\mathscr{P}$.

Thus to show that Cohen-Macaulay local rings of embedding dimension $d, d+1$ or $e+d-1$ have Cohen-Macaulay associated graded rings, it sufficient to show that 1-dimensional local CohenMacaulay rings of embedding dimension 1, 2 or $e$ have Cohen-Macaulay associated graded rings. (However, the usual proof for embedding dimension $d$ or $d+1$ is more direct.) Another application of (2.4) follows in $\S 3$.

As the techniques in the proof of (2.4) have other uses, we put this result in a more general setting. In $\S 1$ we saw that "superregularity" can be expressed in terms of certain intersection equalities. What we need are conditions which allow intersection equalities in dimension $d-1$ to be lifted to dimension $d$.

Proposition 2.1. Let $(R, \underline{m})$ be a local ring and $\underline{x}=x_{1}, \cdots, x_{t}$ be a regular sequence. The following statements are equivalent.

1. $(\underline{x}) \cap \underline{m}^{i+1}=\underline{x}^{i}$ for $i \leqq$ some positive integer $l$.

2. (a) $\left(\underline{m} / x_{1} R\right)^{i+1} \cap\left(\widetilde{x}_{2}, \cdots, \widetilde{x}_{t}\right) R / x_{1} R=\left(\widetilde{x}_{2}, \cdots, \widetilde{x}_{t}\right)\left(\underline{m} / x_{1} R\right)^{i}$ for $i \leqq l$, where $\sim$ denotes image in $R / x_{1} R$;

(b) If $f_{s}$ is a homogeneous polynomial in the polynomial ring $R\left[X_{1}, \cdots, X_{t}\right]$ of (arbitrary) degree $s$ with some coefficient a unit such that $r f_{s}\left(x_{1}, \cdots, x_{t}\right) \in \underline{m}^{i+1}$ for some $r \in R$ and any $i \leqq l$, then $r \in \underline{m}^{i+1-s}$.

3. (a) $\left(\underline{m} / x_{1} R\right)^{i+1} \cap\left(\widetilde{x}_{2}, \cdots, \widetilde{x}_{t}\right) R / x_{1} R=\left(\widetilde{x}_{2}, \cdots, \widetilde{x}_{t}\right)\left(\underline{m} / x_{1} R\right)^{i}$ for $i \leqq l$, where $\sim$ denotes image in $R / x_{1} R$;

(b) $\left(x_{1}\right) \cap \underline{m}^{i+1}=x_{1} \underline{m}^{i}$ for $i \leqq l$.

ExAmPLe. Let $k$ be a field and let $R=k[[X, Y, Z]] /\left(Y^{3}-X Z\right)=$ $k[[x, y, z]]$ with $\underline{m}=(x, y, z) . \quad R$ is Cohen-Macaulay and $x, z$ is a regular sequence. In $R / x R \cong k[[Y, Z]] /\left(Y^{3}\right)$, we have $(m / x R)^{i+1} \cap$ $z R / x R=z(m / x R)^{i}$ for $i \geqq 0$. Similarly, in $R / z R$ we have $(\underline{m} / z R)^{i+1} \cap$ $x R / z R=x(m / z R)^{i}$ for $i \geqq 0$. But $\underline{m}^{3} \cap(x, z) \neq(x, z) \underline{m}^{2}$ as $x z \in \underline{m}^{3}$. Thus the image of $z$ is super-regular in $R / x R$, the image of $x$ is super-regular in $R / z R$ but neither $x$ nor $z$ is super-regular in $R$. 
Proof of 2.1. Suppose (1) holds. (2) (a) follows immediately. We prove (2) (b) by induction on $t$ and on $i+1-s$. There is no difficulty if $t=1$ so we assume $t>1$ and we may assume also that $i+1-s>0$. Let $f_{s}$ and $r$ be as in (2) (b). Let

( $\left.^{*}\right) \quad f_{s}\left(x_{1}, \cdots, x_{t}\right)=a x_{1}^{\alpha_{1}} \cdots x_{t}^{\alpha_{t}}+\cdots+c x_{1}^{\gamma_{1}} \cdots x_{t}^{\gamma_{t}}$,

with $a$ a unit. We may assume that $r$ is not a multiple of any $x_{i}$. If $\alpha_{1}=0$ in (*), by passing to $R / x_{1} R$ where all the hypotheses of (1) hold for the images of $x_{2}, \cdots, x_{t}$, we get $r \in\left(\underline{m}^{i+1-s}, x_{1}\right) . \quad r=\mu+r^{\prime} x_{1}$ with $\mu \in \underline{m}^{i+1-s}$ and $r^{\prime} x_{1} f_{s}\left(x_{1}, \cdots, x_{t}\right) \in \underline{m}^{i+1}$. Since $x_{1} f_{s}$ has degree $s+1$ and $i+1-(s+1)<i+1-s, r^{\prime} \in m^{i+1-(s+1)}$ and $r \in m^{i+1-s}$. Thus we may assume that $x_{1}$ appears in each monomial with unit coefficient in $\left(^{*}\right)$. Pass to the ring $R(U)=R[U]_{m R[U]^{\prime}} U$ an indeterminate and let $x_{1}^{\prime}=x_{1}+U x_{2}$. Since $R \rightarrow R(U)$ is faithfully flat, $x_{1}^{\prime}, x_{2}, \cdots, x_{t}$ is a regular sequence in $R(U)$ and $\left(x_{1}^{\prime}, x_{2}, \cdots, x_{t}\right) \cap(\underline{m} R(U))^{i+1}=$ $\left(x_{1}^{\prime}, x_{2}, \cdots, x_{t}\right)(\underline{m} R(U))^{i}$ for $i \leqq l$. We have

$$
f_{s}\left(x_{1}, \cdots, x_{t}\right)=a\left(x_{1}^{\prime}-U x_{2}\right)^{\alpha_{1}} \cdots x_{t}^{\alpha_{t}}+\cdots+c\left(x_{1}^{\prime}-U x_{2}\right)^{\gamma_{1}} \cdots x_{t}^{\gamma_{t}},
$$

with $r f_{s}\left(x_{1}, \cdots, x_{t}\right) \in(\underline{m} R(U))^{i+1}$. We have

$$
\begin{aligned}
f_{s}\left(x_{1}, \cdots, x_{t}\right)=a x_{1}^{\prime \alpha_{1}} \cdots x_{t}^{\alpha_{t}}+\cdots \\
\quad-a U^{\alpha_{1}} x_{2}^{\alpha_{1}+\alpha_{2}} \cdots x_{t}^{\alpha_{t}}+\cdots-c U^{r_{1}} x_{2}^{\gamma_{1}+\gamma_{2}} \cdots x_{t}^{\gamma_{t}} .
\end{aligned}
$$

Since $f_{s}$ has terms with $x_{1}^{\prime}$ missing, we want to consider $f_{s}$ as a form in $x_{1}^{\prime}, \cdots, x_{t}$ and apply the same argument as above. However, there may be some collapsing in $\left(^{* *}\right)$ so we have to collect terms and write $f_{s}$ as a sum of distinct monominals of degree $s$ in $x_{1}^{\prime}, \cdots, x_{t}$. If all the other monomials in $\left({ }^{* *}\right)$ are distinct from $x_{2}^{\alpha_{1}+\alpha_{2}} \cdots x_{t}^{\alpha_{t}}$ then, by passing to $R(U) / x_{1}^{\prime} R(U)$ and using the same reasoning as above, we see that $r f_{s} \in(\underline{m} R(U))^{i+1}$ implies that $r \in$ $(\underline{m} R(U))^{i+1-s} \cap R=\underline{m}^{i+1-s}$. So we assume there is some collapsing and let $a, a_{i_{1}}, \cdots, a_{i_{g}}$ be unit coefficients in $\left(^{*}\right)$ such that the monomials $x_{2}^{\alpha_{i_{j}}+\alpha_{i_{j}}} \cdots x_{t}^{\alpha_{i}{ }^{t}}$ in $\left({ }^{* *}\right)$ having coefficients $a_{i_{j}} U^{\alpha_{i_{j}}}$ are equal to $\mathscr{H}=$ $x_{2}^{\alpha_{1}+\alpha_{2}} \cdots x_{t}^{\alpha_{t}}$. Note that $x_{2}^{\alpha_{i_{j}}+\alpha_{j^{2}}} \cdots x_{t}^{\alpha_{i} t}=\mathscr{H}$ implies that $\alpha_{i^{3}}=$ $\alpha_{3}, \cdots, \alpha_{i t}=\alpha_{t}$ and $\alpha_{i^{1}}+\alpha_{i^{2}}=\alpha_{1}+\alpha_{2}$. Thus $\alpha_{i^{1}} \neq \alpha_{1}$ since the monomials in $\left(^{*}\right)$ are distinct. Let $b$ be the coefficient (in $\underline{m}$ and possibly zero) of $\mathscr{C}$ in $\left(^{*}\right)$. The new (collected) coefficient of $\mathscr{H}$ is

$$
A_{\mathscr{C}}=-a U^{\alpha_{1}}-a_{i_{1}} U^{\alpha_{i_{1}}}-\cdots-a_{i_{g}} U^{\alpha_{i_{g}}}+b .
$$

Since the powers of $U$ are distinct, $A_{*}$ is a unit. Thus $f_{s}$ may be written as a form of degree $s$ in $x_{1}^{\prime}, x_{2}, \cdots, x_{t}$ with coefficient of the monomial $\mathscr{C}$ a unit. Since $x_{1}^{\prime}$ is missing from $\mathscr{H}$, we apply the same argument as above to show that $r \in \underline{m}^{i+1-s}$. This concludes the proof that $(1) \Rightarrow(2)$. 
Clearly $(2) \Rightarrow(3)$. Suppose (3) holds. If $t=1$, then $j=1$ and (1) holds. Assume $t>1$. Let $w=a x_{1}+g\left(x_{2}, \cdots, x_{t}\right) \in(\underline{x}) \cap \underline{m}^{i+1}$. With $\sim$ denoting images in $R / x_{1} R$, we have $\widetilde{g}\left(\widetilde{x}_{2}, \cdots, \widetilde{x}_{t}\right) \in\left(\widetilde{x}_{2}, \cdots, \widetilde{x}_{t}\right) \cap$ $\left(\underline{m} / x_{1} R\right)^{i+1}=\left(\widetilde{x}_{2}, \cdots, \widetilde{x}_{t}\right)\left(\underline{m} / x_{1} R\right)^{i}$. Thus $g\left(x_{2}, \cdots, x_{t}\right)=h\left(x_{2}, \cdots, x_{t}\right)+$ $x_{1} r$ where $h\left(x_{2}, \cdots, x_{t}\right) \in\left(x_{2}, \cdots, x_{t}\right) m^{i}$. We have $w=a x_{1}+r x_{1}+$ $h\left(x_{2}, \cdots, x_{t}\right) \in \underline{m}^{i+1}$ so $(a+r) x_{1} \in \underline{m}^{i+1} \cap\left(x_{1}\right)=x_{1} \underline{m}^{i}$. Since $x_{1}$ is a nonzero divisor; $a+r \in \underline{m}^{i}$ and $w \in \underline{x} \underline{m}^{i}$.

(2.2) below is the technical result needed to reduce dimension. To eliminate excess notation, homomorphic images of the regular sequence $\underline{x}=x_{1}, \cdots, x_{t}$ will be denoted by the same letters, and if $U_{1}, \cdots, U_{p}$ are indeterminates, we denote

$$
R\left(U_{1}, \cdots, U_{p}\right)=R\left[U_{1}, \cdots, U_{p}\right]_{\underline{m} R\left[U_{1}, \cdots, U_{p}\right]}
$$

by $R(U)$.

THEOREM 2.2. Let $(R, \underline{m})$ be a local ring and let $\underline{x}=x_{1}, \cdots, x_{t}$ be a regular sequence with $t>1$. Suppose that

$$
\left(\underline{m} / x_{1} R\right)^{i+1} \cap\left(x_{2}, \cdots, x_{t}\right) R / x_{1} R=\left(x_{2}, \cdots, x_{t}\right)\left(\underline{m} / x_{1} R\right)^{i}
$$

for $i \leqq$ some positive integer $l$. Suppose that for any finite set of indeterminates $U_{1}, \cdots, U_{p}$, there are elements $x_{i_{1}}, \cdots, x_{i_{p}} \in\left\{x_{2}, \cdots, x_{t}\right\}$ and a linear polynomial $g\left(U_{1}, \cdots, U_{p}\right)=x_{1}+x_{i_{1}} U_{1}+\cdots+x_{i_{p}} U_{p}$ such that

$$
\begin{aligned}
& \left(x_{2}, \cdots, x_{t}\right)(R(U) / g R(U)) \cap(\underline{m} R(U) / g R(U))^{i+1} \\
& \quad=\left(x_{2}, \cdots, x_{t}\right)(\underline{m} R(U) / g R(U))^{i}, \text { for } i \leqq l .
\end{aligned}
$$

Then,

$$
\left(x_{1}, \cdots, x_{t}\right) \cap \underline{m}^{i+1}=\left(x_{1}, \cdots, x_{t}\right) \underline{m}^{i}, \text { for } i \leqq l \text {. }
$$

Proof. We prove that (2)(b) of (2.1) holds for any local ring with a regular sequence satisfying the hypotheses of the theorem by induction on $i+1-s$, where $s, f_{s}$ and $r$ are as in the statement of $(2)(\mathrm{b})$. We may assume that $i+1-s>0$. Let

(*) $\quad f_{s}=a x_{1}^{\alpha_{1}} \cdots x_{t}^{\alpha_{t}}+\cdots+c x_{1}^{\gamma_{1}} \cdots x_{t}^{\gamma_{t}}$, with $a$ a unit .

If $\alpha_{1}=0$, pass to $R / x_{1} R$. By $(2.1)(1) \Rightarrow(2)(\mathrm{b})$, we can conclude that $r \in\left(\underline{m}^{i+1-s}, x_{1}\right) . \quad r=\mu+r^{\prime} x_{1}$ with $\mu \in \underline{m}^{i+1-s}$. But then $r^{\prime} x_{1} f_{s} \in \underline{m}^{i+1}$ and $x_{1} f_{s}$ has degree $s+1$. By induction, we have $r^{\prime} \in \underline{m}^{i+1-(s+1)}$, and $r \in m^{i+1-s}$. Thus we may assume that $x_{1}$ appears in each monomial in $\left(^{*}\right)$ with unit coefficient. By hypothesis, for the indeterminate $U$, there is an element, say $x_{2}$ in $\left\{x_{2}, \cdots, x_{t}\right\}$ such that 


$$
\begin{gathered}
\left(x_{2}, \cdots, x_{t}\right)\left(R(U) / x_{1}^{\prime} R(U)\right) \cap\left(\underline{m} R(U) / x_{1}^{\prime} R(U)\right)^{i+1} \\
=\left(x_{2}, \cdots, x_{t}\right)\left(\underline{m} R(U) / x_{1}^{\prime} R(U)\right)^{i},
\end{gathered}
$$

for $i \leqq l$, where $x_{1}^{\prime}=x_{1}+U x_{2}$.

We have $f_{s}=a\left(x_{1}^{\prime}-U x_{2}\right)^{\alpha_{1}} x_{2}^{\alpha_{2}} \cdots x_{t}^{\alpha_{t}}+\cdots+c\left(x_{1}^{\prime}-U x_{2}\right)^{\gamma_{1}} x_{2}^{\gamma_{2}} \cdots x_{t}^{\gamma_{t} t}$. The same argument as in the proof of $(2.1)(1) \Rightarrow(2)(b)$ shows that $f_{s}$ can be written as a homogeneous polynomial, in the regular sequence $x_{1}^{\prime}, x_{2}, \cdots, x_{t}$, of degree $s$, having a monomial with unit coefficient in which $x_{1}^{\prime}$ does not appear: $f_{s}=A(U) x_{2}^{\eta_{2}} \cdots x_{t}^{\eta_{t}}+\cdots+$ $B(U) x_{1}^{\prime \tau_{1}} x_{2}^{\tau_{2}} \cdots x_{t}^{\tau t}$, with $A(U)$ a unit in $R(U) . \quad r f_{s} \in(m R(U))^{i+1}$. By passing to $R(U) / x_{1}^{\prime} R(U)$ and applying (2.1) (1) $\Rightarrow(2)(\mathrm{b})$, we get $r \in$ $\left((\underline{m} R(U))^{i+1-s}, x_{1}^{\prime}\right)$ so $r=\mu+r^{\prime} x_{1}^{\prime}$ with $\mu \in(\underline{m} R(U))^{i+1-s}$ and $r^{\prime} \in R(U)$. Now $R(U)$ and the regular sequence $x_{1}^{\prime}, x_{2}, \cdots, x_{t}$ satisfy the hypotheses of the theorem. Since $r^{\prime} x_{1}^{\prime} f_{s} \in(\underline{m} R(U))^{i+1}$ and $i+1-(s+1)<$ $i+1-s$, we have by induction that $r^{\prime} \in(\underline{m} R(U))^{i+1-(s+1)}$. Thus $r \in(\underline{m} R(U))^{i+1-s} \cap R=\underline{m}^{i+1-s}$.

We will say that a property $\mathscr{P}$ of a local ring $(R, \underline{m})$ is firm if $\mathscr{P}$ is preserved under the change of rings $R \rightarrow R(U), U$ an indeterminate. We will say that a firm property $\mathscr{P}$ of a $d$-dimensional local Cohen-Macaulay ring $(R, \underline{m})$ is stable for the minimal reduction $\underline{x}=x_{1}, \cdots, x_{d}$ of $\underline{m}$ if for any finite set of indeterminates $U_{1}, \cdots, U_{p}$, $\mathscr{P}$ is preserved under reduction of $R\left(U_{1}, \cdots, U_{p}\right)$ modulo the ideal generated by any subset of generators of $\left(x_{1}, \cdots, x_{d}\right) R\left(U_{1}, \cdots, U_{p}\right)$. We will say that a firm property $\mathscr{P}$ of a $d$-dimensional local CohenMacaulay ring $(R, \underline{m})$ is stable if $\mathscr{P}$ is preserved under reduction modulo the ideal generated by any element of a minimal reduction of $\underline{m}$.

ExAmples. If $(R, \underline{m})$ is a local Cohen-Macaulay ring, the property of being Gorenstein or regular is clearly stable. Let $e$ be a fixed positive integer. The property $\mathscr{P}$ for a local Cohen-Macaulay ring to have multiplicity $e$ is a stable property. As mentioned in the first paragraph of $\S 2$, the property $\mathscr{T}$ for a local Cohen-Macaulay ring of dimension $d$ and multiplicity $e$ to have embedding dimension $e+d-k$ is stable. As example of a property stable for a particular minimal reduction $x$ is given in $\S 3$.

TheOREM 2.3. Let $(R, m)$ be a d-dimensional local CohenMacaulay ring with a property $\mathscr{P}$ stable for the minimal reduction $\underline{x}=x_{1}, \cdots, x_{\dot{a}}$ of $\underline{m}$. Suppose that 1-dimensional local Cohen-Macaulay rings $(S, n)$ with $\mathscr{P}$ satisfy $(x) \cap \underline{n}^{i+1}=x \underline{n}^{i}$ for $i \leqq$ some positive integer $l$ and any minimal reduction $x$ of $\underline{n}$. Then $(\underline{x}) \cap \underline{m}^{i+1}=\underline{x} \underline{m}^{i}$ for $i \leqq l$. 
Proof. The proof is by induction on $d$. We take $d>1$. Let $U=U_{1}, \cdots, U_{p}$ be a finite set of indeterminates. Let $g(U)=x_{1}+$ $U_{1} x_{2}+\cdots+U_{p} x_{2}$. By hypothesis, $R(U) / g(U) R(U)$ has $\mathscr{P}$ and is stable for the minimal reduction $\widetilde{x}_{2}, \cdots, \widetilde{x}_{d}$ which is the image of $x_{2}, \cdots, x_{d}$ in $R(U) / g(U) R(U)$. By induction, $(m R(U) / g(U) R(U))^{i+1} \cap$ $\left(\widetilde{x}_{2}, \cdots, \widetilde{x}_{d}\right)=\left(\widetilde{x}_{2}, \cdots, \widetilde{x}_{d}\right)(\underline{m} R(U) / g(U) R(U))^{i}$ for $i \leqq l$. By (2.2), $m^{i+1} \cap\left(x_{1}, \cdots, x_{d}\right)=\left(x_{1}, \cdots, x_{d}\right) \underline{m}^{i}$ for $i \leqq l$.

Corollary 2.4. Let $(R, \underline{m})$ be a d-dimensional local CohenMacaulay ring with a property $\mathscr{P}$ stable for the minimal reduction $\underline{x}=x_{1}, \cdots, x_{d}$ of $\underline{m}$. If grS is Cohen-Macaulay for every 1-dimensional local Cohen-Macaulay ring $(S, \underline{n})$ with $\mathscr{P}$, then gr $R$ is CohenMacaulay.

For further use it will be convenient to have a variant of (2.3) which requires us to test only some of the minimal reductions in dimension 1. We need the same types of stability for properties of a minimal reduction. Let $(R, \underline{m})$ be a $d$-dimensional local CohenMacaulay ring. Let $U$ be an indeterminate. Let $\underline{x}=x_{1}, \cdots, x_{d}$ be a minimal reduction of $\underline{m}$. Let $Q$ be a property of $\underline{x} R$. We will say that $Q$ is firm if $x R(U)$ has $\mathbb{Q}$. We will say that a firm property $Q$ if stable if, given any element $g$ of a minimal generating set for $\underline{x} R(U)$, say $\underline{x} R(U)=\left(g, y_{2}, \cdots, y_{d}\right) R(U)$, the image $\left(y_{2}, \cdots, y_{d}\right)(R(U) / g R(U))$ has $\mathbb{Q}$.

For example, let $(R, \underline{m})$ be a $d$-dimensional local Cohen-Macaulay ring and, for positive integers $j$, let $\mathscr{Q}_{j}$ be the property for minimal reductions $\underline{x}$ of $\underline{m}$ that $\underline{m}^{j} \leqq \underline{x} R$. Then $\mathscr{Q}_{j}$ is stable. Let $\mathscr{Q}_{j}^{\prime}$ be the property that $\underline{m}^{j} \nsubseteq \underline{x} R$. Then $\mathscr{Q}_{j}^{\prime}$ is also stable.

THeOREM 2.5. Let $(R, \underline{m})$ be a d-dimensional local Cohen-Macaulay ring with a property $\mathscr{\mathscr { P }}$ stable for the minimal reduction $\underline{x}=$ $x_{1}, \cdots, x_{d}$ of $\underline{m}$. Assume in addition that $\underline{x}$ has a stable property Q. Suppose that 1-dimensional local Cohen-Macaulay rings $(S, \underline{n})$ with $\mathscr{P}$ satisfy $(x) \cap \underline{n}^{i+1}=x \underline{n}^{i}$ for $i \leqq$ some positive integer $l$ and all minimal reductions $x$ of $\underline{n}$ which have the stable property $\mathbb{Q}$. Then $(\underline{x}) \cap \underline{m}^{i+1}=\underline{x} \underline{m}^{i}$ for $i \leqq l$.

Proof. The proof is the same as the proof of (2.3).

\section{Applications.}

THEOREM 3.1. Let $(R, \underline{m})$ be a d-dimensional local Gorenstein ring of multiplicity $e$ and embedding dimension $e+d-3$. If $\underline{x}=$ $x_{1}, \cdots, x_{d}$ is any minimal reduction of $\underline{m}, \underline{m}^{3} \nsubseteq \underline{x} R$ and $\underline{m}^{4} \subset \underline{x} \underline{m}$. 
$g r R$ is Cohen-Macaulay if and only if $\underline{m}^{4}=\underline{x} \underline{m}^{3}$ for some minimal reduction $x$ of $m$.

Proof. Let $\underline{x}=x_{1}, \cdots, x_{d}$ be a minimal reduction of $\underline{m}$. Pass to $(\widetilde{R}, \underline{\tilde{m}})=(R / \underline{x} R, \underline{m} / \underline{x} R)$. Since $v(\underline{\tilde{m}})=e-3$ and $\widetilde{\lambda}(\widetilde{R})=e$, we must have that $\underline{\tilde{m}}^{4}=0$. Since $\lambda($ socle $\widetilde{R})=1, \underline{\widetilde{m}}^{3}=$ socle $\widetilde{R}$. Thus $\underline{m}^{3} \nsubseteq \underline{x} R$ and $\underline{m}^{4} \subset \underline{x} R$. By the analytic independence of $\underline{x}, \underline{m}^{4} \subset \underline{x} m$.

If, for some minimal reduction $\underline{x}$ of $\underline{m}, \underline{m}^{4} \nsubseteq \underline{x}^{3}$, then $\bar{x}_{1}, \cdots, \bar{x}_{d}$ is a homogeneous system of parameters in $g r R$ that is not a regular sequence by (1.1) so that $g r R$ is not Cohen-Macaulay by [2].

To complete the proof we will show that if $(R, \underline{m})$ is a $d$-dimensional local Cohen-Macaulay ring of embedding dimension $e+d-3$ and if $\underline{x}$ is a minimal reduction of $\underline{m}$ such that $\underline{m}^{3} \nsubseteq \underline{x} R$ and $\underline{m}^{4}=$ $\underline{x} \underline{m}^{3}$ then $g r R$ is Cohen-Macaulay. Let $\mathscr{P}$ be the property for $d$ dimensional local Cohen-Macaulay rings $(S, \underline{n})$ that $S$ has embedding dimension $e+d-3$ and that there exists a minimal reduction $\underline{y}$ of $\underline{n}$ such that $\underline{n}^{3} \nsubseteq \underline{y} R$ and $\underline{n}^{4}=y \underline{n}^{3} . \quad R$ has $\mathscr{P}$ and $\mathscr{P}$ is stable for the minimal reduction $\underline{x}$ of $\underline{m}$. Thus by (2.4), we may assume $d=1$. Let $\underline{x}=x$ and let $\underline{m}=\left(x, w_{1}, \cdots, w_{e-3}\right)$. There exist $p, q \in$ $\{1, \cdots, e-3\}$ such that $\underline{m}^{2}=\left(x \underline{m}, w_{p} w_{q}\right), \underline{m}^{3}=\left(x \underline{m}^{2}, w_{p}^{2} w_{q}\right)$ and $w_{p}^{2} w_{q} \notin$ $x \underline{m}$. This follows from the fact that $(\underline{m} / x R)^{2}$ and $(\underline{m} / x R)^{3}$ are nonzero principal ideals. It is clear that $\underline{m}^{i+1} \cap(x)=x \underline{m}^{i}$ for $i \geqq 3$. We must show that $\underline{m}^{3} \cap(x)=x \underline{m}^{2}$. Let $z \in \underline{m}^{3} \cap(x) . \quad z=x \mu+r w_{p}^{2} w_{q}$ with $\mu \in \underline{m}^{2}$ and $r \in R$. Since $r w_{p}^{2} w_{q} \in(x), r \in \underline{m}$ and $r w_{p}^{2} w_{q} \in \underline{m}^{4}=x \underline{m}^{3}$. Thus $z \in x \underline{m}^{2}$ and $\underline{m}^{3} \cap(x)=x \underline{m}^{2}$.

REMARKS. Note that, with the hypotheses of (3.1), if $g r R$ is Cohen-Macaulay it is not Gorenstein. (3.1) may also be proved directly, using just (1.5) instead of (2.4) and (1.5).

EXAMPLes. 1. Let $k$ be a field. The rings $k\left[\left[t^{5}, t^{6}, t^{9}\right]\right]$, $k\left[\left[t^{6}, t^{7}, t^{10}, t^{11}\right]\right], k\left[\left[t^{6}, t^{11}, t^{13}, t^{20}\right]\right]$ are Gorenstein and satisfy $v=e+d-3$ and $\underline{m}^{4}=x \underline{m}^{3}$. Recall that the multiplicity $e$ of a numerical semigroup ring $R=k\left[\left[t^{\alpha_{1}}, t^{\alpha_{2}}, \cdots, t^{\alpha_{n}}\right]\right]$ with $\alpha_{1}<\alpha_{2}<\cdots<\alpha_{n}$ and $\operatorname{gcd}\left(\alpha_{1}, \alpha_{2}, \cdots, \alpha_{n}\right)=1$ is just $\alpha_{1}$. This follows for example, from [8, VIII, $\S 10$, Thm. 24].

2. $k\left[\left[t^{5}, t^{6}, t^{9}\right]\right]=k[[X, Y, Z]] /\left(X^{3}-Y Z, Y^{3}-Z^{2}\right)$ is a complete intersection with associated graded ring $k[X, Y, Z] /\left(Y Z, Z^{2}, Y^{4}-Z X^{3}\right)$ which is Cohen-Macaulay but not a complete intersection.

3. The hypothesis that $R$ is Gorenstein cannot be omitted from (3.1). Let $R=k\left[\left[t^{7}, t^{8}, t^{11}, t^{13}, t^{17}\right]\right]$, with $k$ a field. $R$ satisfies $v(\underline{m})=$ $e+d-3=7+1-3$ and $\underline{m}^{4}=t^{7} \underline{m}^{3}$ but $g r R$ is not Cohen-Macaulay at $t^{17} \underline{m} \subset \underline{m}^{3}$.

It is possible that the hypothesis $\underline{m}^{4}=\underline{x} \underline{m}^{3}$ is redundant in (3.1), 
i.e., it is possible that all $d$-dimensional local Gorenstein rings of embedding dimension $e+d-3$ have Cohen-Macaulay associated graded rings. ${ }^{1}$ By (2.4) it is sufficient to prove this for $d=1$. It is true that if $(R, \underline{m})$ is a 1-dimensional analytically irreducible local Gorenstein domain with algebraically closed residue field and with embedding dimension $e+d-3$, then $g r R$ is Cohen-Macaulay.

Next, we want to show that Cohen-Macaulay local rings with certain Hilbert polynomials have Cohen-Macaulay associated graded rings. First, we recall definitions of the concepts involved. For any $d$-dimensional local ring $(R, \underline{m})$, the Hilbert function is defined for nonnegative integers $n$ by $H_{R}(n)=\operatorname{dim}_{R / \underline{m}}\left(\underline{m}^{n} / \underline{m}^{n+1}\right)$. The Hilbert sum transforms are defined inductively for nonnegative integers $n$ by

$$
H_{i 2}^{0}(n)=H_{R}(n) \quad \text { and } \quad H_{R}^{i}(n)=\sum_{j=0}^{n} H_{R}^{i-1}(j) .
$$

For large $n, H_{R}^{i}(n)$ is a polynomial $P_{R}^{i}(n)$ of degree $d-1+i$. If $x \in \underline{m}$, then $H_{R / x R}^{1}(n)-H_{R}^{0}(n)=\lambda\left(\left(\underline{m}^{n+1}: x\right) / \underline{m}^{n}\right)$, cf. [8], Lemma 3 , VIII, $\S 8$. If $x$ is super-regular, $H_{R / x R}^{1}(n)=H_{R}^{0}(n)$. If $x$ just has the property that $\left(\underline{m}^{n+1}: x\right)=\underline{m}^{n}$ for all large $n$, we still get that $P_{R / x R}^{1}(n)=P_{R}^{\lrcorner}(n)$. This prompts the definition of superficial element, cf. [8], VIII, §8. An element $x$ in a local ring $(R, \underline{m})$ is superficial if there is a positive integer $c$ such that $\left(\underline{m}^{n+1}: x\right) \cap \underline{m}^{c}=\underline{m}^{n}$, for all $n \geqq c$. If $x$ is a superficial element and a nonzero divisor then $\left(\underline{m}^{n+1}: x\right)=\underline{m}^{n}$ for all large $n$. A superficial element is the preimage in $R$ of an element of degree 1 in $g r R$ which does not lie in any prime belonging to 0 in $g r R$ except possibly $g r m$. Superficial elements exist if $R / \underline{m}$ is infinite. If, in addition, $\underline{m}$ does not belong to 0 in $R$, there exists a superficial element which is also a nonzero divisor.

Theorem 3.2. Let $(R, m)$ be a d-dimensional local CohenMacaulay ring with $d \geqq 2$ and multiplicity $e$. The Hilbert polynomial $P_{R}^{0}(n)$ for $R$ is

$$
P_{R}^{0}(n)=\left(\begin{array}{c}
n+d-2 \\
n-1
\end{array}\right) e+\left(\begin{array}{c}
n+d-2 \\
n
\end{array}\right)
$$

if and only if $R$ has embedding dimension $e+d-1$ in which case grR is Cohen-Macaulay.

Proof. It was proved in [4] that $v(\underline{m})=e+d-1$ implies that $g r R$ is Cohen-Macaulay and in [5] that $v(\underline{m})=e+d-1$ implies that $H_{R}^{\circ}(n)=\left(\begin{array}{c}n+d-2 \\ n-1\end{array}\right) e+\left(\begin{array}{c}n+d-2 \\ n\end{array}\right)$, for all $n \geqq 0$.

Suppose that $(R, \underline{m})$ is a local Cohen-Macaulay ring with Hilbert polynomial $P_{R}^{\circ}(n)=\left(\begin{array}{c}n+d-2 \\ n-1\end{array}\right) e+\left(\begin{array}{c}n+d-2 \\ n\end{array}\right)$. We will show

\footnotetext{
${ }^{1}$ Added in proof. The author has recently verified that this is the case.
} 
that $v(\underline{m})=e+d-1$ by induction on $d$. Let $d=2$. We may assume that $R / \underline{m}$ is infinite and take a superficial element $x$ with $x$ a nonzero divisor. Then, for $n \geqq n_{0}$, some integer $n_{0}, H_{R / x R}^{1}(n)=$ $H_{R}^{0}(n)=n e+1 . \quad R / x R$ is a 1-dimensional local Cohen-Macaulay ring so, for all $n \geqq 1, H_{R / x R}^{1}(n)=1+e-j_{1}+e-j_{2}+\cdots+e-j_{n}$ with nonnegative integers $j_{i}$ having the property that if $j_{i}=0$, then $j_{k}=0$ for $k \geqq i$. Since for $n \geqq n_{0}, H_{R / x R}^{1}(n)=1+n e-\sum_{k=1}^{n} j_{k}=$ $1+n e$, it must be true that $\sum_{k=1}^{n} j_{k}=0$. Thus $v(\underline{m} / x R)=e$ and $v(\underline{m})=e+1$.

Suppose $d>2$. Again, assuming $R / \underline{m}$ infinite, if necessary, we take a superficial element $x$ which is also a nonzero divisor. For large $n, H_{R / x R}^{1}(n)=H_{R}^{0}(n)=\left(\begin{array}{c}n+d-2 \\ n-1\end{array}\right) e+\left(\begin{array}{c}n+d-2 \\ n\end{array}\right)$. Since $H_{R / x R}^{0}(n)=H_{R / x R}^{1}(n)-H_{R / x R}^{1}(n-1)$, we have for large $n$,

$$
\begin{aligned}
H_{R / x R}^{0}(n)= & \left(\begin{array}{c}
n+d-2 \\
n-1
\end{array}\right) e+\left(\begin{array}{c}
n+d-2 \\
n
\end{array}\right) \\
& -\left(\begin{array}{c}
n-1+d-2 \\
n-2
\end{array}\right) e-\left(\begin{array}{c}
n-1+d-2 \\
n-1
\end{array}\right) \\
= & \left(\begin{array}{c}
n+(d-1)-2 \\
n-1
\end{array}\right) e+\left(\begin{array}{c}
n+(d-1)-2 \\
n
\end{array}\right) .
\end{aligned}
$$

Thus, by induction, $v(\underline{m} / x R)=e+(d-1)-1$. Therefore, $v(\underline{m})=$ $e+d-1$.

REMARK. Clearly, the hypothesis $d \geqq 2$ is necessary in (3.2) as every 1-dimensional local Cohen-Macaulay ring $(R, \underline{m})$ has $P_{R}^{\circ}(n)=e$.

TheOREM 3.3. Let $(R, m)$ be a d-dimensional local CohenMacaulay ring with $d \geqq 2$, and multiplicity $e$. The Hilbert polynomial $P_{R}^{0}(n)$ for $R$ is

$$
P_{R}^{o}(n)=\left(\begin{array}{c}
n+d-2 \\
n-1
\end{array}\right) e+\left(\begin{array}{c}
n+d-3 \\
n
\end{array}\right)
$$

if and only if $R$ has embedding dimension $e+d-2$ and $g r R$ is Cohen-Macaulay.

We need two lemmas. Lemma 3.4 was given in [6]. We give a proof below using the results of $\S 1$.

LEMma 3.4. Let $(R, \underline{m})$ be a d-dimensional local Cohen-Macaulay ring of multiplicity $e$ and embedding dimension $e+d-2$. Let $\underline{x}=x_{1}, \cdots, x_{d}$ be a minimal reduction for $\underline{m}$. Then $\underline{m}^{3} \subset(\underline{x})$ and $g r R$ is Cohen-Macaulay if and only if $\underline{m}^{3}=\underline{x}^{2}$, for some minimal 
reduction $\underline{x}$ of $\underline{m}$.

Proof. Pass to $(\widetilde{R}, \underline{\widetilde{m}})=(R / \underline{x} R, \underline{m} / \underline{x} R) . \quad \lambda(\widetilde{R})=e$ and $v(\underline{\tilde{m}})=$ $e-2$. Counting lengths, we see that $\underline{\tilde{m}}^{2} \neq 0$ and $\underline{\tilde{m}}^{3}=0$. Thus $\underline{\widetilde{m}}^{3} \subset(\underline{x})$. Suppose that $\underline{m}^{3}=\underline{x} \underline{m}^{2}$. Then $\underline{m}^{i+1} \cap(\underline{x})=\underline{x} \underline{m}^{i}$ for $i \geqq 2$. But, by the analytic independence of $\underline{x}$, cf. [3], we also have $\underline{m}^{2} \cap$ $(\underline{x})=\underline{x} \underline{m}$. Thus $g r R$ is Cohen-Macaulay by (1.5). If, on the other hand, $\underline{m}^{3} \nsubseteq x \underline{m}^{2}$, then by (1.1) there is some $j, 1 \leqq j \leqq d$, such that $\left(\bar{x}_{1}, \cdots, \bar{x}_{j-1}: \bar{x}_{j}\right) \nsubseteq\left(\bar{x}_{1}, \cdots, \bar{x}_{j-1}\right)+(g r \underline{m})^{2}$ so that $g r R$ is not Cohen-Macaulay.

Lemma 3.5. Let $(R, \underline{m})$ be a d-dimensional local Cohen-Macaulay ring with $d \geqq 2$. Let $\underline{x}=x_{1}, \cdots, x_{d}$ be a minimal reduction for $\underline{m}$. Suppose that $\left(\underline{m} / x_{1} R\right)^{3} \cap\left(x_{2}, \cdots, x_{d}\right) R / x_{1} R=\left(x_{2}, \cdots, x_{d}\right)\left(\underline{m} / x_{1} R\right)^{2}$ and that $\left(\underline{m} / x_{2} R\right)^{3} \cap\left(x_{1}, x_{3}, \cdots, x_{d}\right) R / x_{2} R=\left(x_{1}, x_{3}, \cdots, x_{d}\right)\left(\underline{m} / x_{2} R\right)^{2}$. Then

$$
\underline{m}^{3} \cap\left(x_{1}, \cdots, x_{d}\right) R=\left(x_{1}, \cdots, x_{d}\right) \underline{m}^{2} \text {. }
$$

Proof. Let $w=a_{1} x_{1}+\cdots+a_{d} x_{d} \in \underline{m}^{3} \cap\left(x_{1}, \cdots, x_{d}\right)$. Passing to $R / x_{1} R$ and $R / x_{2} R$ in turn, we get that $a_{1}, a_{2}, \cdots, a_{d} \in\left(\underline{m}^{2}, \underline{x}\right)$. Thus $w=f_{2}\left(x_{1}, \cdots, x_{d}\right)+w^{\prime}$, with $w^{\prime} \in\left(x_{1}, \cdots, x_{d}\right) \underline{m}^{2}$ and $f_{2}\left(x_{1}, \cdots, x_{d}\right)$ a form of degree 2 in $x_{1}, \cdots, x_{d} . f_{2}\left(x_{1}, \cdots, x_{d}\right) \in \underline{m}^{3}$, by the analytic independence of $\underline{x}, f_{2}\left(x_{1}, \cdots, x_{d}\right) \in(\underline{x})^{2} \underline{m}$ and $w \in \underline{x} \underline{m}^{2}$, as desired.

Proof of 3.3. The proof is by induction on $d$. We may assume that $R / \underline{m}$ is infinite and take a minimal reduction $\underline{x}=x_{1}, \cdots, x_{d}$ of $m$ having the property that each $x_{i}$ is a superficial element. Let $d=2$. For large $n$ and for $i=1,2, H_{R / x_{i} R}^{1}(n)=H_{R}^{0}(n)=n e$. Since $R / x_{i} R$ is a 1 -dimensional local Cohen-Macaulay ring, for every $n \geqq 1$, $H_{R / x_{i} R}^{1}(n)=1+e-j_{1}+\cdots+e-j_{n}$, with nonnegative integers $j_{k}$ having the property that if $j_{l}=0$, then $j_{k}=0$ for $k \geqq l$. For large $n, H_{R / x_{i} R}^{1}(n)=1+n e-\sum_{k=1}^{n} j_{k}=n e$. Thus, $\sum_{k=1}^{n} j_{k}=1$ so $j_{1}=1$ and $j_{k}=0$ for $k>1$. It follows that $v\left(\underline{m} / x_{i} R\right)=e-1$ and $v\left(\left(\underline{m} / x_{i} R\right)^{l}\right)=e$ for $l>1$. Therefore, $v(\underline{m})=e$ and $\left(\underline{m} / x_{1} R\right)^{3}=x_{2}\left(\underline{m} / x_{1} R\right)^{2}$ and $\left(\underline{m} / x_{2} R\right)^{3}=x_{1}\left(\underline{m} / x_{2} R\right)^{2}$. By $(3.5)$, we have $\underline{m}^{3} \cap\left(x_{1}, x_{2}\right)=\left(x_{1}, x_{2}\right) \underline{m}^{2}$ and by (3.4), $g r R$ is Cohen-Macaulay.

Assume that $d>2$. For each $x_{i}, i=1, \cdots, d$, and for large $n$, we have $H_{R / x_{i} R}^{1}(n)=H_{R}^{0}(n)=\left(\begin{array}{c}n+d-2 \\ n-1\end{array}\right) e+\left(\begin{array}{c}n+d-2 \\ n\end{array}\right)$. Since, $H_{R / x_{i} R}^{0}(n)=H_{R / x_{i} R}^{1}(n)-H_{R / x_{1} R}^{1}(n-1)$, it follows that

$$
H_{R / x_{i} R}^{0}(n)=\left(\begin{array}{c}
n+(d-1)-2 \\
n-1
\end{array}\right) e+\left(\begin{array}{c}
n+(d-1)-2 \\
n
\end{array}\right),
$$

for large $n$. We may apply induction to $R / x_{i} R$ to get $v\left(\underline{m} / x_{i} R\right)=$ 
$e+(d-1)-2$ and $v(\underline{m})=e+d-2$. Also, by induction, $\left(\underline{m} / x_{i} R\right)^{3}=$ $\left(x_{1}, \cdots, x_{i}, \cdots, x_{d}\right)\left(\underline{m} / x_{i} R\right)^{2}$, so, by (3.5) and (3.4), $\underline{m}^{3}=\left(x_{1}, \cdots, x_{d}\right) \underline{m}^{2}$ and $g r R$ is Cohen-Macaulay.

REMARK. (3.3) answers a question D. Mumford asked the author.

\section{REFERENCES}

1. N. Bourbaki, Éléments de Mathematique, Fasc. XXVIII, Algèbre Commutative, Hermann, Paris, 1967.

2. M. Hochster and J. L. Ratliff, Jr., Five theorems on Macaulay rings, Pacific J. Math., 44 (1973), 147-172.

3. D. C. Northcott and D. Rees, Reductions of ideals in local rings, Proc. Camb. Phil. Soc., 50 (1954), 145-158.

4. J.D. Sally, On the associated graded ring of a local Cohen-Macaulay ring, J. Math. Kyoto Univ., 17 (1977), 19-21.

5. — Cohen-Macaulay local rings of maximal embedding dimension, J. of Algebra, 56 (1979), 168-183.

6. - Tangent cones at Gorenstein singularities, Comp. Math., to appear.

7. B. Singh, Effect of a permissible blowing-up on the local Hilbert functions, Inv. Math., 26 (1974), 201-212.

8. O. Zariski and P. Samuel, Commutative Algebra II, D. Van Nostrand, Princeton, 1960.

Received July 5, 1978 and in revised form January 19, 1979. The author is grateful to the Sloan Foundation and to the National Science Foundation for support during the preparation of this work.

NORTHWESTERN UNIVERSITY

Evanston, IL 60201 



\section{PACIFIC JOURNAL OF MATHEMATICS}

\section{EDITORS}

DONALD BABBITT (Managing Editor)

University of California

Los Angeles, CA 90024

HUGO RossI

University of Utah

Salt Lake City, UT 84112

C. C. Moore and ANDrew OGG

University of California

Berkeley, CA 94720
J. DUGUNDJI

Department of Mathematics University of Southern California Los Angeles, CA 90007

R. FINN and J. MILGRAM

Stanford University

Stanford, CA 94305

\section{ASSOCIATE EDITORS}
E. F. BeCKENBACH
B. H. NeumanN
F. WOLF
K. YOSHIDA

\section{SUPPORTING INSTITUTIONS}

UNIVERSITY OF BRITISH COLUMBIA CALIFORNIA INSTITUTE OF TECHNOLOGY UNIVERSITY OF CALIFORNIA MONTANA STATE UNIVERSITY UNIVERSITY OF NEVADA, RENO NEW MEXICO STATE UNIVERSITY OREGON STATE UNIVERSITY UNIVERSITY OF OREGON

\author{
UNIVERSITY OF SOUTHERN CALIFORNIA \\ STANFORD UNIVERSITY \\ UNIVERSITY OF HAWAII \\ UNIVERSITY OF TOKYO \\ UNIVERSITY OF UTAH \\ WASHINGTON STATE UNIVERSITY \\ UNIVERSITY OF · WASHINGTON
}

The Supporting Institutions listed above contribute to the cost of publication of this Journal, but they are not owners or publishers and have no responsibility for its content or policies.

Mathematical papers intended for publication in the Pacific Journal of Mathematics should be in typed form or offset-reproduced, (not dittoed), double spaced with large margins. Please do not use built up fractions in the text of the manuscript. However, you may use them in the displayed equations. Underline Greek letters in red, German in green, and script in blue. The first paragraph or two must be capable of being used separately as a synopsis of the entire paper. Please propose a heading for the odd numbered pages of less than 35 characters. Manuscripts, in triplicate, may be sent to any one of the editors. Please classify according to the scheme of Math. Reviews, Index to Vol. 39. Supply name and address of author to whom proofs should be sent. All other communications should be addressed to the managing editor, or Elaine Barth, University of California, Los Angeles, California, 90024.

50 reprints to each author are provided free for each article, only if page charges have been substantially paid. Additional copies may be obtained at cost in multiples of 50 .

The Pacific Journal of Mathematics is issued monthly as of January 1966. Regular subscription rate: $\$ 84.00$ a year (6 Vols., 12 issues). Special rate: $\$ 42.00$ a year to individual members of supporting institutions.

Subscriptions, orders for numbers issued in the last three calendar years, and changes of address should be sent to Pacific Journal of Mathematics, P.O. Box 969, Carmel Valley, CA 93924, U.S.A. Older back numbers obtainable from Kraus Periodicals Co., Route 100, Millwood, NY 10546.

PUBLISHED BY PACIFIC JOURNAL OF MATHEMATICS, A NON-PROFIT CORPORATION

Printed at Kokusai Bunken Insatsusha (International Academic Printing Co., Ltd.). 8-8, 3-chome, Takadanobaba, Shinjuku-ku, Tokyo 160, Japan.

Copyright (C) 1979 by Pacific Journal of Mathematics Manufactured and first issued in Japan 


\section{Pacific Journal of Mathematics}

\section{Vol. 84, No. $2 \quad$ June, 1979}

Somesh Chandra Bagchi and Alladi Sitaram, Spherical mean periodic

functions on semisimple Lie groups ........................ 241

Billy Joe Ball, Quasicompactifications and shape theory............. 251

Maureen A. Bardwell, The o-primitive components of a regular ordered permutation group ................................ 261

Peter W. Bates and James R. Ward, Periodic solutions of higher order

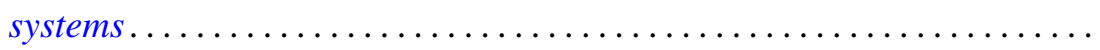

Jeroen Bruijning, A characterization of dimension of topological spaces by totally bounded pseudometrics......................... 283

Thomas Farmer, On the reduction of certain degenerate principal series

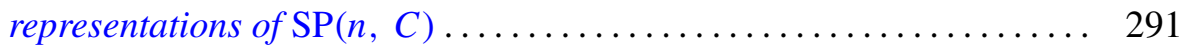

Richard P. Jerrard and Mark D. Meyerson, Homotopy with m-functions . . . . 305

James Edgar Keesling and Sibe Mardesic, A shape fibration with fibers of

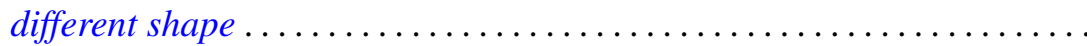

Guy Loupias, Cohomology over Banach crossed products. Application to bounded derivations and crossed homomorphisms ...............

Rainer Löwen, Symmetric planes ........................ 367

Alan L. T. Paterson, Amenable groups for which every topological left

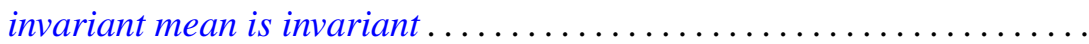

Jack Ray Porter and R. Grant Woods, Ultra-Hausdorff H-closed extensions

Calvin R. Putnam, Operators satisfying a $G_{1}$ condition .

Melvin Gordon Rothenberg and Jonathan David Sondow, Nonlinear smooth representations of compact Lie groups..............

Werner Rupp, Riesz-presentation of additive and $\sigma$-additive set-valued measures.

A. M. Russell, A commutative Banach algebra of functions of generalized variation

Judith D. Sally, Superregular sequences

Patrick Shanahan, On the signature of Grassmannians . . 